\title{
Loss of glomerular foot processes is associated with uncoupling of podocalyxin from the actin cytoskeleton
}

\author{
Tetsuro Takeda, ${ }^{1}$ Tammie McQuistan, ${ }^{1}$ Robert A. Orlando ${ }^{2}$ and Marilyn G. Farquhar ${ }^{1,2}$ \\ ${ }^{1}$ Department of Cellular and Molecular Medicine, and \\ ${ }^{2}$ Department of Pathology, University of California, San Diego, La Jolla, California, USA
}

Address correspondence to: Marilyn Gist Farquhar, Department of Cellular and Molecular Medicine, University of California, San Diego, 9500 Gilman Drive, La Jolla, California 92093-0651, USA.

Phone: (858) 534-7711; Fax: (858) 534-8549; E-mail: mfarquhar@ucsd.edu.

Tetsuro Takeda's present address is: Division of Clinical Nephrology and Rheumatology,

Niigata University Graduate School of Medical and Dental Sciences, Niigata, Japan.

Robert A. Orlando's present address is: Department of Biochemistry and Molecular Biology,

University of New Mexico Health Sciences Center, Albuquerque, New Mexico, USA.

Received for publication February 16, 2001, and accepted in revised form June 6, 2001.

\begin{abstract}
Podocalyxin (PC), the major sialoprotein of glomerular epithelial cells (GECs), helps maintain the characteristic architecture of the foot processes and the patency of the filtration slits. PC associates with actin via ezrin, a member of the ERM family of cytoskeletal linker proteins. Here we show that $\mathrm{PC}$ is linked to ezrin and the actin cytoskeleton via $\mathrm{Na}^{+} / \mathrm{H}^{+}$-exchanger regulatory factor 2 (NHERF2), a scaffold protein containing two PDZ (PSD-95/Dlg/ZO-1) domains and an ERM-binding region. The cytoplasmic tail of PC contains a C-terminal PDZ-binding motif(DTHL) that binds to the second PDZ domain of NHERF2 in yeast two-hybrid and in vitro pull-down assays. By immunocytochemistry NHERF2 colocalizes with PC and ezrin along the apical domain of the GEC plasma membrane. NHERF2 and ezrin form a multimeric complex with PC, as they coimmunoprecipitate with PC. The PC/NHERF2/ezrin complex interacts with the actin cytoskeleton, and this interaction is disrupted in GECs from puromycin aminonucleoside-, protamine sulfate-, or sialidase-treated rats, which show a dramatic loss of foot processes, comparable to that seen in the nephrotic syndrome. Thus NHERF2 appears to function as a scaffold protein linking PC to ezrin and the actin cytoskeleton. PC/NHERF2/ezrin/actin interactions are disrupted in pathologic conditions associated with changes in GEC foot processes, indicating their importance for maintaining the unique organization of this epithelium.
\end{abstract}

J. Clin. Invest. 108:289-301 (2001). DOI:10.1172/JCI200112539.

\section{Introduction}

Although it has been over 40 years since the first description of the dramatic changes in the organization of the glomerular epithelium cells (GECs) that occurs in the nephrotic syndrome $(1,2)$, the molecular mechanisms involved are still obscure (3-5). It was recognized early that GECs possess highly negative charged, sialic acid-rich cell surface components that bind cationic dyes by histochemical staining $(6,7)$ and that the changes seen in the nephrotic syndrome are linked to the surface charge of the GECs, because similar changes in podocyte cell shape with foot process flattening and rearrangement of cell junctions can be induced by neutralization of their surface charge with protamine sulfate $(8-10)$ or by removal of sialic acid $(11,12)$. Some time ago (13), we showed that the surface charge on GEC is carried for the most part by podocalyxin (PC), an integral membrane protein on the apical surface of GEC that has the distribution and biochemical properties characteristic of the histochemically defined glomeru- lar polyanion $(6,7)$, and we proposed that $\mathrm{PC}$ functions in the maintenance of the unique foot process and filtration slit architecture of GECs. More recently, we directly demonstrated that PC functions as an antiadhesin responsible for keeping the filtration slits open and that removal of sialic acid from PC affects cell-cell adhesion and junctional permeability (14). How neutralization of the surface charge on the ectodomain of PC mediates changes in GEC organization remains unclear. We demonstrated recently that the cytoplasmic domain of PC is linked to the actin cytoskeleton by ezrin (15), a member of the ERM (ezrin/radixin/moesin) family, which is concentrated on the cytoplasmic side of the apical domain of the plasma membrane (PM) along the cell bodies and foot processes of GECs (16). ERM proteins are typically located peripherally along the PM and link the cytoplasmic tails of PM proteins to the cortical actin cytoskeleton. They play a role in the formation of microvilli, cell-cell junctions, and membrane ruffles as well as substrate adhesion and mobility $(17,18)$. 
The involvement of PC in maintaining the normal glomerular filtration barrier and its linkage to the actin cytoskeleton suggested that this molecule might also be involved in mediating the changes in foot process organization that occur in the nephrotic syndrome. Previously, we demonstrated that the expression of PC correlates with differentiation of foot processes and filtration slits (19), and others showed that in puromycin aminonucleoside (PAN) nephrosis, a rat model of nephrosis, there is a change in the distribution of actin filaments within GECs (20). These findings led us to investigate whether the association of $\mathrm{PC}$ with the actin cytoskeleton may be altered in disease models associated with changes in GEC architecture.

In this study we demonstrate that $\mathrm{Na}^{+} / \mathrm{H}^{+}$-exchanger regulatory factor 2 (NHERF2), a PDZ (PSD-95/ $\mathrm{Dlg} / \mathrm{ZO}-1$ ) protein, interacts with the C-terminus of PC through its second $\mathrm{PDZ}$ domain, forms a complex with ezrin, and links the complex to the actin cytoskeleton. Furthermore, we find that in situations where the foot process and slit organization are altered, e.g., in rats treated with PAN, protamine sulfate, or sialidase, there are disruptions in the PC/NHERF2/ezrin complex that links PC to the actin cytoskeleton. Thus we provide direct evidence that $\mathrm{PC}$ plays a role in maintaining the unique cell architecture of GECs through interaction with the actin cytoskeleton, which is mediated by NHERF2 and ezrin.

\section{Methods}

Materials. Enhanced chemiluminescent substrate (SuperSignal) was obtained from Pierce Chemical Co. (Rockford, Illinois, USA). Redivue $\left[{ }^{35} \mathrm{~S}\right]$ methionine $(>1,000 \mathrm{Ci} / \mathrm{mmol})$ and Redivue $\left[{ }^{32} \mathrm{P}\right] \mathrm{dCTP}$ were from Amersham Pharmacia Biotech (Piscataway, New Jersey, USA). Puromycin aminonucleoside, protamine sulfate (grade X), heparin (grade IA), and sialidase (type III, from Vibrio cholerae) were obtained from Sigma-Aldrich (Milwaukee, Wisconsin, USA). Primers were synthesized by Life Technologies Inc. (Gaithersburg, Maryland, USA), and restriction enzymes were purchased from New England Biolabs Inc. (Beverly, Massachusetts, USA). Chemical reagents were obtained from Sigma-Aldrich except as indicated.

Animals. Male Sprague-Dawley rats (150-250 g) were obtained from Harlan Bioproducts for Science Inc. (Indianapolis, Indiana, USA). Animals were kept in an air-conditioned room and maintained on a commercial stock diet and tap water ad libitum.

Construction of the rat glomerular cDNA library and yeast two-bybrid screening. Rat glomeruli were isolated by graded sieving as described previously (13). Double-stranded cDNA was generated from $5 \mu \mathrm{g}$ poly $(\mathrm{A})^{+} \mathrm{RNA}$ purified from rat glomeruli using a random-primed cDNA synthesis kit according to the manufacturer's instructions (Life Technologies Inc.). EcoRI-adaptors were ligated onto the cDNA at the $5^{\prime}$ and $3^{\prime}$ end, and the library was inserted into the B42 activation domain of the displayTARGET "prey" vector (Display Systems Biotech,
Vista, California, USA). The total library contains approximately $2 \times 10^{5}$ independent clones. The cDNA encoding the entire cytoplasmic domain of rat PC (amino acids 411-485) (14) was amplified by PCR and inserted into the EcoRI and XhoI sites in the LexA DNAbinding domain of "bait" vector. The resulting bait vector, PBAIT-PCT, and the reporter vector (containing the green fluorescence protein gene as a reporter) were transformed into yeast strain, displayYEAST-L.

For interaction screening in the LexA-based version of the yeast two-hybrid system, $20 \mu \mathrm{g}$ of the rat glomerular cDNA library in the prey vector was transformed into the yeast, which was pretransformed with pBAIT-PCT and reporter vectors. Yeast transformants $5 \times 10^{5}$ were plated onto selective medium (-histidine, -uracil, -leucine, -glucose, +galactose), and surviving colonies were exposed to standard ultraviolet (UV) light $(360 \mathrm{~nm})$. Twenty-eight transformants grew up and showed green fluorescence using UV light. Positive clones were grouped by restriction enzyme digestion analysis, and seven inserts of different sizes were selected. Plasmid DNA from the positive colonies was rescued by electroporation into Escherichia coli KC8 (CLONTECH Laboratories Inc., Palo Alto, California, USA) and retransformed back into the yeast containing original pBAIT-PCT plasmid and various control plasmids for one-to-one interactions. Four of the seven positives interacted only with the pBAIT-PCT and not with a nonspecific pBAIT-p53. Both strands of the DNA inserts were sequenced by automated sequencing (Molecular Pathology Shared Resource, University of California, San Diego, Cancer Center, La Jolla, California, USA).

Database searches and sequence analysis. Basic alignment search tool (BLAST) searches were performed at the Web site of the National Center for Biotechnology Information (21). Amino acid alignments and sequence analysis were performed using MacVector version 6.5.3 software (Accelyrs Corp., San Diego, California, USA). cDNA cloning. To obtain the complete coding sequence for rat NHERF2, $5^{\prime}$ rapid amplification of cDNA ends (RACE) was performed on the same rat glomerular cDNA library with the primer (5'-GGTCCCCTTCGTAAGTGGCAGAGC-3') designed from the sequence obtained as described previously (14). The RACE product $(\sim 1.2 \mathrm{~kb})$ was ligated into pGEM-T Easy vector. Three clones of the RACE product were sequenced on both strands.

Plasmid constructs. Rabbit NHERF1 cDNA and human NHERF2 cDNA were kindly provided by Randy Hall (Duke University, Durham, North Carolina, USA). To generate the N-terminal FLAG-tag construct pNF, we inserted the ds-oligonucleotides encoding the FLAG epitope (DYKDDDDK) into the HindIII and BamHI sites of PCDNA3. PDZ domains from NHERF1 and NHERF2 were generated by PCR using rabbit NHERF1 and rat NHERF2 cDNA as templates (Figure 1). We also expressed clone A19 (amino acid 140-337), one of the partial clones initially obtained from yeast twohybrid screening, which contains the second PDZ 
domain and the C-terminal ERM-binding region (Figure 1). The PCR products (N1P1, N2P1, N1P2, and $\mathrm{N} 2 \mathrm{P} 2$ ) and the insert from NHERF1, NHERF2, and clone $\mathrm{A} 19$ were subcloned into $\mathrm{pNF}$ to produce $\mathrm{pNF}-$ N1P1, pNF-N2P1, pNF-N1P2, pNF-N2P2, pNFNHERF1, pNF-NHERF2, and pNF-N2P2-C, respectively. The cDNA of PSD95 was also subcloned into pNF. The PC expression vector, PIRES-PC, was constructed earlier (14). pIRES-PC( $\triangle \mathrm{DTHL})$, which lacks the putative PDZ domain-binding motif (DTHL), was generated from pIRES-PC by PCR. Fidelity of the PCR products was confirmed by automated sequencing.

In vitro pull-down assays. The cDNA encoding the cytoplasmic domain of rat PC (amino acid 411-485) was amplified by PCR and inserted into pGEX-KG (Amersham Pharmacia Biotech), and glutathione S-transferase (GST) fusion proteins or GST alone was expressed in E. coli DH5 $\alpha$, purified, and immobilized on glutathione-agarose beads (Amersham Pharmacia Biotech) as described by the manufacturer. ${ }^{35}$ S-labeled full-length NHERF1, NHERF2, or various domains from each NHERF were produced by TNT T7 rabbit reticulocyte Quick Coupled Transcription/Translation system (Promega Corp., Madison, Wisconsin, USA) in the presence of $\left[{ }^{35} \mathrm{~S}\right]$ methionine and various $\mathrm{pNF}$ constructs according to the manufacturer's instructions. In vitro translated products were incubated with approximately $7 \mu \mathrm{g}$ GST-PC cytoplasmic tail (GST-PCT) or approximately $5 \mu \mathrm{g}$ GST alone (equivalent to the amount of GST-PCT) immobilized on glutathioneagarose beads in binding buffer (20 mM HEPES, $\mathrm{pH} 7.4$, $150 \mathrm{mM} \mathrm{NaCl}, 2 \mathrm{mM} \mathrm{CaCl} 2,10 \mathrm{mM}$ CHAPS, $1 \mathrm{mM}$ DTT, $0.1 \% \mathrm{BSA}$, and $10 \mu \mathrm{g} / \mathrm{ml}$ leupeptin) for 15 hours at $4^{\circ} \mathrm{C}$ with gentle rocking. Beads were then washed (four times) with the same buffer without BSA, and bound proteins were eluted with $25 \mu \mathrm{l}$ Laemmli sample buffer, resolved by $15 \%$ SDS-PAGE, and visualized by autoradiography with Biomax MR x-ray film (Eastman Kodak Co., Rochester, New York, USA).

$A b$ 's. Polyclonal rabbit anti-PC (0601) raised against the cytoplasmic domain of PC (14) and anti-PC mAbs $1 \mathrm{~A}$ and $5 \mathrm{~A}$ that recognize the ectodomain of rat $\mathrm{PC}(22)$ were prepared as described previously. Affinity-purified polyclonal anti-EBP50 IgG (also called NHERF1) was provided by Anthony Bretscher (Cornell University, Ithaca, New York, USA). Polyclonal anti-E3KARP sera (also called NHERF2) was obtained from Chris Yun (Johns Hopkins University, Baltimore, Maryland, USA), anti-NHERF2 mAb from Pann-Ghill Suh (Pohang University, Pohang, South Korea), and polyclonal antiezrin from Heinz Furthmayr (Stanford University, Palo Alto, California, USA). An mAb, 297S, specific for ezrin, radixin, and moesin phosphorylated on threonine residues 567, 564, or 558, respectively (23), was provided by Sachiko Tsukita (Kyoto University, Kyoto, Japan). Anti-ezrin (3C12), anti-actin (AC-40), and anti-FLAG (M2) mAbs were purchased from Sigma-Aldrich. Crossadsorbed Alexa 488-conjugated goat anti-rabbit and Alexa 594-conjugated goat anti-mouse $\mathrm{F}\left(\mathrm{ab}^{\prime}\right)_{2}$ were purchased from Molecular Probes Inc. (Eugene, Oregon, USA). Five nanometer and $10 \mathrm{~nm}$ gold goat antirabbit or anti-mouse IgG conjugates were from Amersham Pharmacia Biotech. Affinity-purified goat anti-rabbit and anti-mouse IgG conjugated to horseradish peroxidase (HRP) were from Bio-Rad Laboratories Inc. (Hercules, California, USA).

SDS-PAGE and immunoblotting. Protein concentration was determined by BCA assay (Pierce Chemical Co.). Proteins were separated on 7,10 , or $12 \%$ SDS-PAGE under reducing conditions and transferred to PVDF membranes (Millipore Corp., Bedford, Massachusetts, USA) using a wet tank transfer system (Minigel-Transfer-Unit; Bio-Rad Laboratories Inc.) at $75 \mathrm{~mA}$ for 15 hours. Membranes were blocked (1 hour in PBS, pH 7.2 , containing $5 \%$ calf serum and $0.1 \%$ Tween- 20 ), incubated for 2 hours at room temperature with primary Ab's, followed by HRP-conjugated goat anti-rabbit or anti-mouse IgG (1-hour incubation, 1:3000 dilution), and detection by enhanced chemiluminescence. Quantification of protein bands was done by densitometry with ScanAnalysis software (Alltech Associates Inc., Deerfield, Illinois, USA).

Cell culture and transfection. COS7 cells were maintained in DMEM high glucose supplemented with $10 \%$ FCS, $100 \mathrm{U} / \mathrm{ml}$ penicillin $\mathrm{G}$, and $100 \mu \mathrm{g} / \mathrm{ml}$ streptomycin sulfate (Life Technologies Inc.). COS7 cells were plated at $3 \times 10^{5}$ cells per $60-\mathrm{mm}$ plate, cultured overnight, and transfected ( $2 \mu \mathrm{g}$ cDNA) using $12 \mu$ l Fugene 6 (Roche Molecular Biochemicals, Indianapolis, Indiana, USA). After 24 hours, the transfected cells were lysed in lysis buffer (0.5\% Triton X-100, 20 mM HEPES, pH 7.5, 150 $\mathrm{mM} \mathrm{NaCl}, 1 \mathrm{mM}$ EDTA, $0.1 \% \mathrm{BSA})$ containing protease inhibitors ( $10 \mu \mathrm{g} / \mathrm{ml}$ leupeptin and $0.5 \mathrm{mM} \mathrm{PMSF})$, and centrifuged at $15,000 \mathrm{~g}$ at $4^{\circ} \mathrm{C}$ for 30 minutes.

Coimmunoprecipitation. Cell lysates $(\sim 200 \mu \mathrm{g})$ were cleared by incubation with $20 \mu \mathrm{l}$ protein $\mathrm{G}$ plus/protein A-agarose beads (Calbiochem-Novabiochem Corp., San Diego, California, USA) for 1 hour, and the supernatant was incubated with $2 \mu \mathrm{g}$ anti-FLAG (M2), $4 \mu \mathrm{g}$ monoclonal anti-PC $(1 \mathrm{~A}+5 \mathrm{~A}), 2 \mu \mathrm{l}$ polyclonal anti-NHERF2, $2 \mu \mathrm{l}$ polyclonal anti-ezrin, or $2 \mu \mathrm{l}$ normal rabbit serum at $4^{\circ} \mathrm{C}$ for 15 hours. Twenty microliters Protein $\mathrm{G}$ PLUS/Protein A-agarose beads were added, and the beads were washed (three times) with the same lysis buffer without BSA and once with PBS. Bound immune complexes were eluted by incubating the beads in Laemmli sample buffer at $95^{\circ} \mathrm{C}$ for 5 minutes and separated by SDS-PAGE followed by immunoblotting.

Preparation of kidneys for immunofluorescence and immunoelectron microscopy. Kidneys were perfused with DMEM followed by $4 \%$ paraformaldehyde (PFA) in $100 \mathrm{mM}$ phosphate buffer, $\mathrm{pH} 7.4$, for 15 minutes, then excised and immersion fixed in 4\% PFA (45 minutes) and 8\% PFA (15 minutes). Samples were cryoprotected and frozen in liquid nitrogen (24). For immunofluorescence, semithin cryosections $(0.5-1.0 \mu \mathrm{m})$ were cut with a Leica Ultracut UCT microtome equipped with an FCS cryoattachment at $-100^{\circ} \mathrm{C}$, transferred to gelatin-coated microscope slides, 


\section{a}
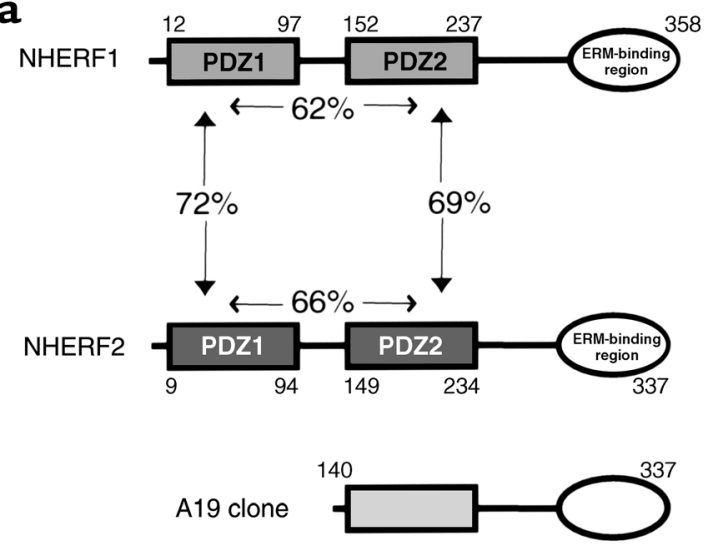

b

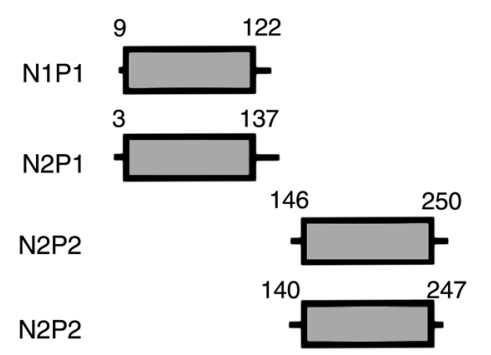

Figure 1

Scheme of the domain organization of the NHERF family and various constructs used in this study. (a) NHERF1 and NHERF2 contain two PDZ domains and a C-terminal ERM-binding region. PDZ domains of NHERF2 share $60-70 \%$ identity with each other and with the equivalent domains of NHERF1. A19 is the partial clone of NHERF2 initially obtained from yeast two-hybrid screening using PC tail as bait. (b) PDZ domains of NHERF proteins prepared by in vitro translation or expressed as FLAG-tagged fusion proteins. N1P1, first PDZ domain of NHERF1; N2P1, first PDZ domain of NHERF2; N1P2, second PDZ domain of NHERF1; N2P2, second PDZ domain of NHERF2.

incubated sequentially at room temperature with primary Ab's (2 hours) and cross-adsorbed Alexa 488-conjugated goat anti-rabbit or Alexa 594-conjugated goat anti-mouse $\mathrm{F}\left(\mathrm{ab}^{\prime}\right)_{2}(1$ hour) (16), and examined with a Zeiss Axiophot microscope (Carl Zeiss Inc., Thornwood, New York, USA). Images were captured with a Cohen CCD camera using Scion image version 1.59 (Scion Corp., Frederick, Massachusetts, USA) and processed using Adobe Photoshop 5.0 (Adobe Systems Inc., San Jose, California, USA). For immunogold labeling, ultrathin cryosections $(80 \mathrm{~nm})$ were cut and processed as described (24), incubated at room temperature with primary Ab's ( 2 hours), followed by 5 or $10 \mathrm{~nm}$ gold-conjugated goat anti-rabbit or mouse IgG (1 hour). Sections were stained for 20 minutes in $2 \%$ ( $\mathrm{vol} / \mathrm{vol})$ neutral uranyl acetate, adsorption stained, and embedded in $0.1 \%$ uranyl acetate $/ 0.2 \%$ methyl-cellulose $/ 3.2 \%$ (vol/vol) polyvinyl alcohol.

For routine electron microscopy (EM), kidneys were flushed with cold $\left(4^{\circ} \mathrm{C}\right)$ HBSS through the abdominal aorta and cut into small blocks $\left(1 \mathrm{~mm}^{3}\right)$, which were immersed in Karnovsky's fixative $(1.5 \%$ glutaraldehyde $/ 3 \%$ paraformaldehyde in $0.1 \mathrm{M}$ cacodylate buffer plus 5\% sucrose, $\mathrm{pH} 7.4$ ) for 1 hour at room temperature, postfixed in $1 \% \mathrm{OsO}_{4}$ for 1 hour at $4^{\circ} \mathrm{C}$, and embedded in epoxy resin (10).

Perfusion with protamine sulfate and sialidase. Male rats $(200-250 \mathrm{~g})$ were anesthetized with Metofane (methoxyflurane), and both kidneys were perfused in situ through the abdominal aorta at a pressure of approximately $100 \mathrm{mmHg}$ and an infusion rate of 6-12 $\mathrm{ml} / \mathrm{min}$ as described previously with slight modifications $(9,25)$. Initially, the kidneys were flushed with HBSS at $37^{\circ} \mathrm{C}$ for 2 minutes, after which protamine sulfate (PS; $500 \mu \mathrm{g} / \mathrm{ml}$ in HBSS) or Vibrio cholerae sialidase (SA; $50 \mathrm{mU} / \mathrm{ml}$ in $\mathrm{HBSS}$ ) were perfused at $37^{\circ} \mathrm{C}$ for 15 or 60 minutes, respectively. The kidneys remained immersed in a water bath at $37^{\circ} \mathrm{C}$ during the entire incubation period. Some animals were perfused with heparin (PS/Hep; $100 \mu \mathrm{g} / \mathrm{ml}$ in HBSS) at $37^{\circ} \mathrm{C}$ for 15 minutes after perfusion with PS (8). Four rats were used for each experimental group.

Induction of PAN nephrosis. Male rats (150 g) were injected once intraperitoneally with PAN $(15 \mathrm{mg} / 100 \mathrm{~g} / \mathrm{rat})$ as described previously (26). Animals were sacrificed on the third (PAN 3d) or tenth (PAN 10d) day after injection. Urinary protein was monitored with Albustix (Miles Inc., Elkhart, Indiana, USA). All animals exhibited proteinuria $(4+)$ and massive ascites on the tenth day but were not proteinuric on the third day.

Differential detergent extraction of glomeruli. For each experimental group glomeruli were isolated by graded sieving at $4^{\circ} \mathrm{C}$ in the presence of protease inhibitors (complete; Roche Molecular Biochemicals). The resultant fractions consisted of more than $90 \%$ glomeruli (13). A differential detergent extraction protocol was used to sequentially solubilize the proteins as described previously with slight modifications (27). First, samples were lysed in $1 \mathrm{ml}$ Triton lysis buffer $(0.5 \%$ Triton $\mathrm{X}-100,20 \mathrm{mM}$ HEPES, $\mathrm{pH}$ 7.5, $150 \mathrm{mM} \mathrm{NaCl}, 1 \mathrm{mM}$ EDTA, $0.5 \mathrm{mM}$ PMSF, $10 \mu \mathrm{g} / \mathrm{ml}$ leupeptin), and centrifuged at $15,000 \mathrm{~g}$ at $4^{\circ} \mathrm{C}$ for 30 minutes. Next, the insoluble pellet was resuspended in the same volume of RIPA buffer $(0.1 \%$ SDS, $0.5 \%$ deoxycholate, $1 \%$ Triton $\mathrm{X}-100,20 \mathrm{mM}$ HEPES, $\mathrm{pH} 7.5,150 \mathrm{mM} \mathrm{NaCl}, 1 \mathrm{mM}$ EDTA, $0.5 \mathrm{mM}$ PMSF, $10 \mu \mathrm{g} / \mathrm{ml}$ leupeptin) and centrifuged as above. The resultant pellet was solubilized by sonication in the same volume of Laemmli sample buffer as the supernatant, and equal volumes of the fractions were analyzed by immunoblotting.

\section{Results}

Identification of NHERF2 as a PC-interacting protein. To identify proteins that interact with $\mathrm{PC}$, we carried out two-hybrid screening of a rat glomerular cDNA library using the cytoplasmic tail of PC as bait. Of the approximately $5 \times 10^{5}$ yeast transformants screened, four clones were confirmed to be positive and required the presence of bait construct for reporter activity. All four clones were sequenced and found to be fragments of the rat ortholog of human NHERF2 (also named E3KARP). The largest clone, A19, extended from the 


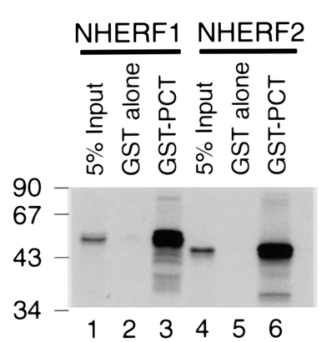

b

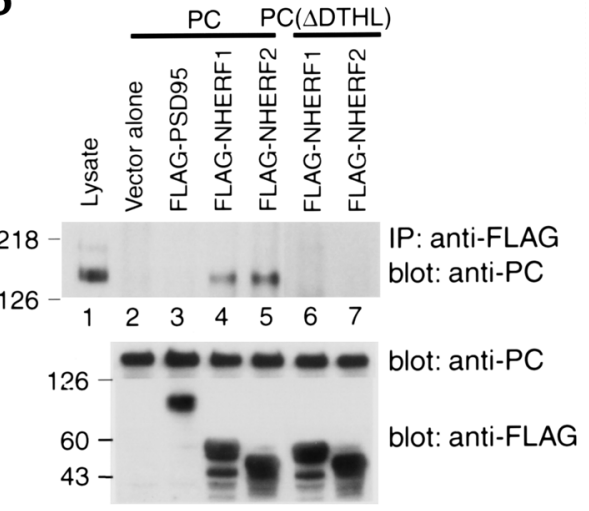

\section{Figure 2}

PC associates with the second PDZ domain of NHERF proteins through its C-terminal PDZ-binding motif. (a) Interaction of GST-PCT with NHERF proteins. NHERF2 bind specifically to GST-PCT but not to GST alone (lanes 2 and 5), as well as NHERF1 (lane 3). Equivalent amounts of GSTPCT $(7 \mu \mathrm{g})$ or GST alone $(\sim 5 \mu \mathrm{g})$ bound to glutathione-agarose beads were incubated with in vitro-translated, radiolabeled NHERF1 or NHERF2. The precipitates were separated by $15 \%$ SDS-PAGE and detected by autoradiography. $5 \%$ input, $5 \%$ of the in vitro-translated products. (b) PC binds NHERF proteins, and the C-terminus of $\mathrm{PC}$ is essential for the interaction. PC coimmunoprecipitates with both NHERF proteins (lanes 4 and 5 ), but not with vector alone (lane 2 ) or with PSD95 (lane 3). PC, lacking the last four C-termi-
C
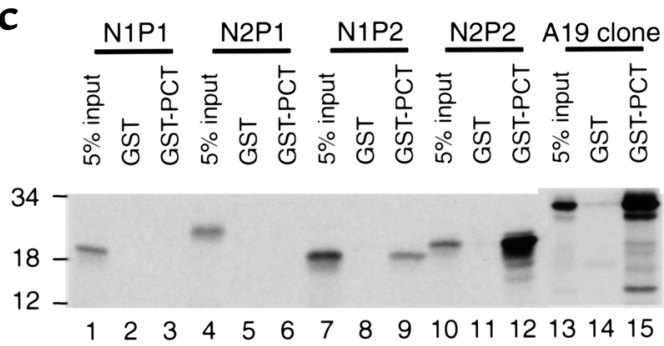

nal residues $[P C(\triangle D T H L)]$, does not associate with either NHERF protein (lanes 6 and 7). Lane 1: $10 \mu \mathrm{g}$ cell lysate. PC or PC $\triangle D T H L)$ were transiently coexpressed in Cos 7 cells with either NHERF1, NHERF2, or PSD95 (control) followed by immunoprecipitation with anti-FLAG (M2). The precipitated proteins were immunoblotted with anti-PC (0601). The middle and lower panels demonstrate that the transfected cells express PC, PC mutant, and NHERF at comparable levels. (c) PDZ2 of both NHERF proteins specifically associates with GST-PCT (lanes 9 and 12). The A19 clone (see Figure 1a) is used as a positive control. Neither PDZ1 domain (lanes 3 and 6) associates with GST-PCT. Experiment performed as in a. Size markers indicate molecular mass in kilodaltons. second PDZ domain to the C-terminal region (Figure 1a). To obtain the complete coding sequence we carried out $5^{\prime}$ RACE on the same cDNA library and obtained a product $(\sim 1.2 \mathrm{~kb})$ covering the entire NHERF2 cDNA with an ORF encoding 337 amino acid (aa). Human NHERF2 was initially cloned by Yun et al. and shown to be involved in the cAMP-dependent protein kinase A regulation of $\mathrm{Na}^{+} / \mathrm{H}^{+}$exchanger 3 (NHE3) of the renal brush border (28). NHERF2 contains two PDZ domains that share $61-72 \%$ identity with each other and to the equivalent domains of NHERF1 (Figure 1a). PDZ domains are modules mediating protein-protein interaction $(29,30)$ and often recognize the C-terminal consensus sequence $(\mathrm{X}(\mathrm{S} / \mathrm{T}) \mathrm{X}(\mathrm{V} / \mathrm{L} / \mathrm{I})$ of target proteins (31). PC has the consensus sequence of a PDZ-binding motif at its C-terminus (DTHL).

$P C$ interacts with NHERF1 and NHERF2. To confirm the interaction of PC with NHERF2 we performed in vitro GST pull-down assays. In vitro-translated NHERF2 bound strongly to GST-PCT but not to GST alone (Figure 2a, lane 6). Given the high homology between NHERF1 and NHERF2, we also tested NHERF1 and found that it also interacted with GSTPCT (Figure 2a, lane 3).

To confirm that interaction between PC and NHERF takes place in vivo, we cotransfected COS7 cells with expression plasmids encoding rat PC and FLAG-tagged NHERF1 or NHERF2 and carried out immunoprecipitation with anti-FLAG antibodies followed by immunoblotting for PC. PC coimmunoprecipitated with both NHERF1 and NHERF2, but not with vector alone or with PSD95, another PDZ protein (Figure 2b, upper panel, lanes 2-5). These results indicate that the cytoplasmic tail of PC specifically interacts with both NHERF proteins in these assays.

To determine whether the extreme C-terminus of PC containing the PDZ-binding motif mediates its association with NHERF proteins, pIRESPC $(\triangle D T H L)$, a PC construct that lacks the last four C-terminal residues, was cotransfected into COS7 cells together with NHERF1 or NHREF2. The PC mutant did not coimmunoprecipitate with either NHERF protein (Figure 2b, upper panel, lanes 6 and 7), indicating that the interaction occurs through the C-terminus of PC.

PC interacts preferentially with PDZ2 of NHERF2. To find out which PDZ domain binds PC, we performed GST pull-down assays with various in vitro-translated fragments of NHERF1 or NHERF2 (see Figure 1b). Fragments containing the second PDZ domain (PDZ2) of both NHERF1 (Figure 2c, lane 9) and NHERF2 (Figure $2 \mathrm{c}$, lane 12) bound to GST-PCT, but binding of PDZ2 of NHERF2 was much greater than PDZ2 of NHERF1. Neither of the PDZ1 domains bound to GST-PCT in vitro. We also carried out yeast two-hybrid assays with the same fragments of NHERF1 and NHERF2 and confirmed the above results (data not shown).

NHERF2 is expressed in glomerular epithelial cells. We next examined whether NHERF1 and NHERF2 codistribute with PC. We showed previously that PC is located exclusively along the apical surface of GECs and glomerular and peritubular capillary endothelia (13). Quite recent- 
ly, NHERF1 was shown to be localized mainly in the brush border region of proximal tubules (32), and NHERF2 was shown to be present in glomeruli and principal cells of collecting ducts, but its distribution within the glomerulus was not determined (33). We compared the distribution of NHERF1, NHERF2, and PC in organ lysates by immunoblotting and found, as expected, that the two NHERF proteins showed different distributions. NHERF1 was highly expressed in the brain and kidney and barely detectable in the liver and lung (Figure 3; ref. 34 ), whereas NHERF2 was highly expressed in the kidney and lung but was barely detectable in the brain. Neither NHERF protein was detected in the heart or spleen under these conditions. NHERF1 was more highly expressed in whole kidney than in glomerular extracts, whereas NHERF2 was highly enriched in glomerular extracts. Thus the distribution of NHERF2, but not NHERF1, is similar to that of PC (Figure 3), being highly enriched in the lung and glomeruli.

By immunofluorescence carried out on semithin cryosections of rat kidney cortex, NHERF2 staining was most concentrated along the apical plasma membrane (PM) of GECs (Figure 4, a and d) where it colocalized with PC. It was not detected in endothelial cells of peritubular or glomerular capillaries that also express PC (Figure 4, $\mathrm{c}$ and $\mathrm{f}$ ). In contrast, NHERF1 was expressed mainly in the brush border region of proximal tubules (Figure 4g) as reported previously (32).

Results obtained by EM after immunogold labeling of ultrathin cryosections confirmed and extended the results obtained by immunofluorescence. After double labeling for NHERF2 and PC, the two proteins colocalized along the apical cell membrane of the cell bodies of GECs and the tops and the sides of the foot processes (Figure $5 \mathrm{a}$ ).

PC and NHERF2 are associated in situ. To determine whether NHERF2 and PC are associated in situ, immunoprecipitation was carried out on glomerular lysates with anti-PC or anti-NHERF2, followed by immunoblotting of the immunoprecipitates for the presence of NHERF2 and PC, respectively. PC was found in the NHERF2 immunoprecipitates (Figure 6a, lane 3); similarly, NHERF2 was present in PC immunoprecipitates (Figure 6b, lane 3). In contrast, NHERF1 was not present in immunoprecipitates obtained with anti-PC (Figure 6b, lane 3), and PC was not present in immunoprecipitates obtained with anti-NHERF1 from whole kidney lysates (data not shown). These results, taken together with our localization studies, clearly indicate that NHERF2 but not NHERF1 interacts with PC in GECs.

Localization of PC and NHERF2 with ezrin in GECs. We recently showed that ezrin colocalizes with $\mathrm{PC}$ along the apical PM of GECs and can be coimmunoprecipitated with PC from cortical lysates, suggesting that it may serve to link PC to the actin cytoskeleton (15). NHERF2 has also been shown to associate with ezrin in vitro (35, 36 ) and in lung lysates (23). These findings raise the possibility that NHERF2 may be associated with both PC and ezrin in GECs. To address this issue, we first examined whether ezrin colocalizes with NHERF2 and whether it associates with NHERF2 in GECs in situ. In semithin cryosections, NHERF2 staining (Figure 4, j and $\mathrm{m}$ ) largely overlaps with ezrin (Figure $4, \mathrm{k}$ and $\mathrm{n}$ ) in glomeruli, but not in proximal tubules. By immunogold labeling, NHERF2, and ezrin colocalize along the apical PM of GECs (Figure 5d), indicating that PC, NHERF2 and ezrin codistribute along the apical PM of GECs. Very little if any staining for either NHERF or ezrin is detected at the base of the foot processes where they attach to the glomerular basement membrane (GBM).

PC, NHERF2, and ezrin form a coimmunoprecipitable complex in glomeruli. To investigate whether PC, NHERF2, and ezrin interact in vivo, we carried out coimmunoprecipitation assays with anti-PC on glomerular lysates followed by immunoblotting for NHERF2 and ezrin. We found that both ezrin and NHERF2 - but not NHERF1 - were present in the immunoprecipitates (Figure 6b, lane 3). Taken together, our results indicate that PC, NHERF2, and ezrin associate in a multimeric complex along the apical PM of GEC.

The PC/NHERF2/Ezrin complex is partially disrupted in glomeruli from PS- and SA-treated kidneys. It was shown previously that dramatic alterations in GECs can be induced by SA treatment $(11,12)$ or by neutralization of the glomerular surface charge with polycations such as PS (8-10). These changes mimic those seen in the nephrotic state in humans or in experimental PAN nephrosis in rats where there is loss of foot processes, collapse of filtration slits, and apical displacement of slit diaphragms (37). Therefore, we examined whether or not the PC/NHERF2/ezrin complexes are disrupted in glomeruli after these treatments. Toward this

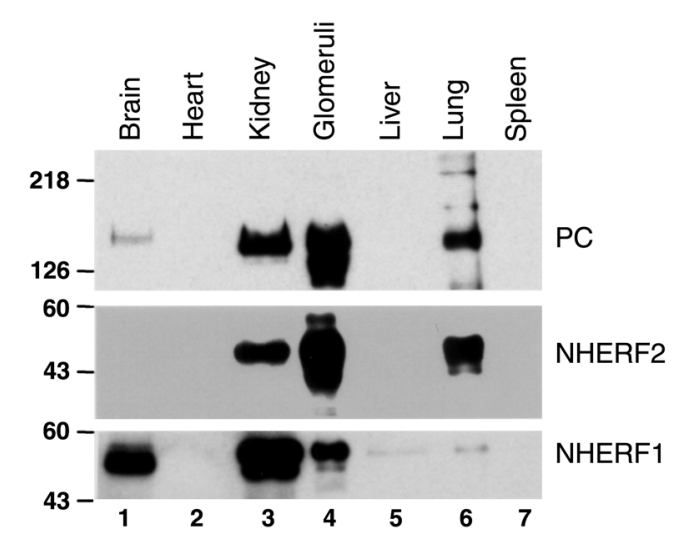

Figure 3

Distribution of PC, NHERF1, and NHERF2. PC and NHERF2 are expressed at high levels in the kidney and lung and are enriched in glomeruli. They are also detected in the brain, but not in heart, liver, or spleen. NHERF1 is present at highest levels in whole kidney and brain, but is not enriched in glomeruli. It is also present at very low levels in the liver and lung, but is not detected in heart and spleen. Ten micrograms of total protein from the indicated rat tissues was separated by $10 \%$ SDS-PAGE, transferred to PVDF membranes, and blotted with polyclonal anti-PC (0601), anti-NHERF1, or anti-NHERF2. 

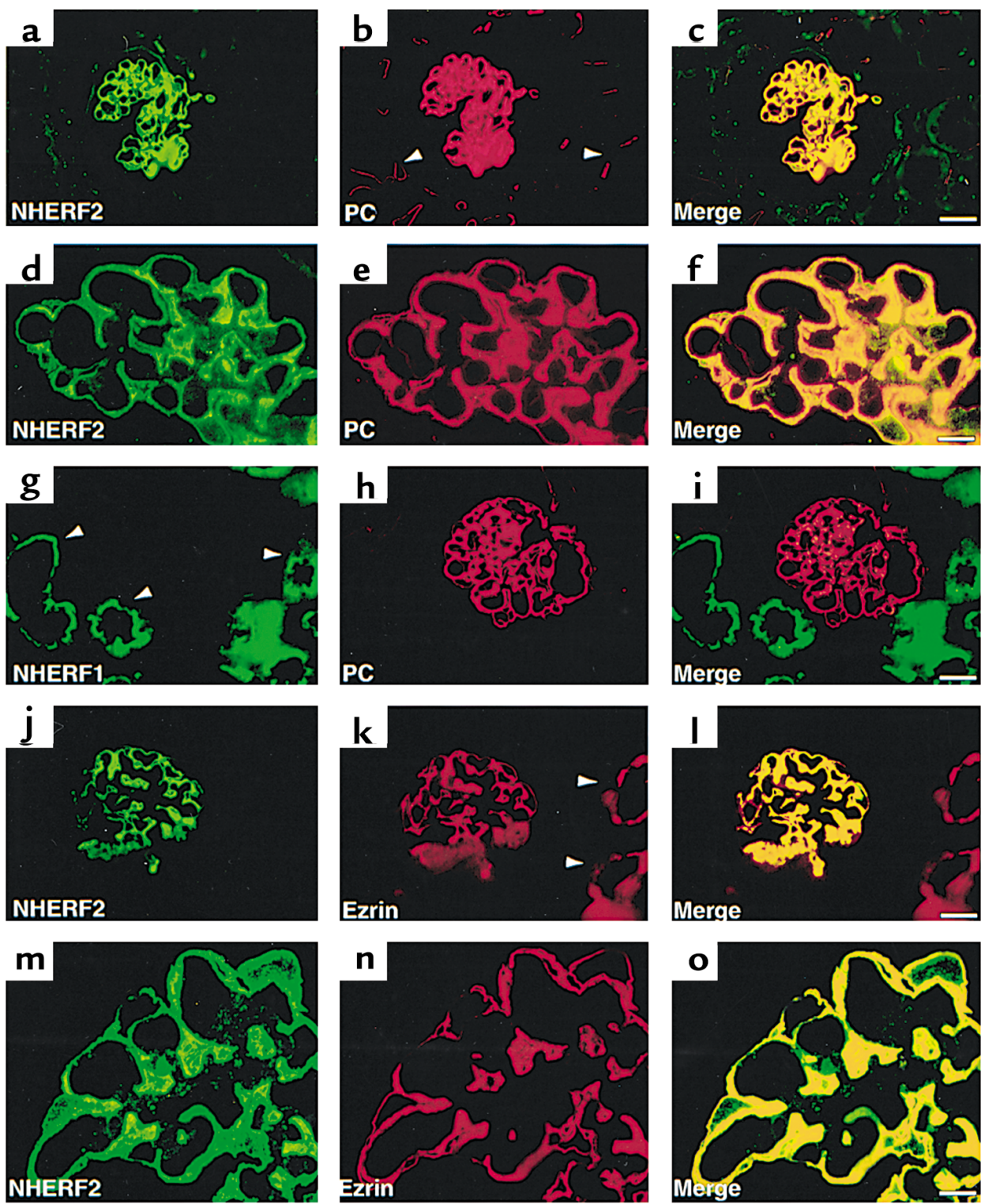

Figure 4

Localization of NHERF1, NHERF2, PC, and ezrin in the normal rat kidney. Strong staining for both NHERF2 (a and d) and PC (b and e) is seen in GECs in the glomerulus. PC is also present in the endothelium of peritubular capillaries (b, arrowhead) where NHERF2 is not seen. The yellow signal in the merged images ( $\mathbf{c}$ and $\mathbf{f}$ ) demonstrate overlap in the staining of GECs. In contrast, NHERF1 (g) is expressed mainly in the apical region of proximal tubules (arrowheads) and is not seen in glomeruli where PC is present (h and $\mathbf{i})$. NHERF2 (j and $\mathbf{m}$ ) also colocalizes with ezrin ( $\mathbf{k}$ and $\mathbf{n}$ ) in GECs where their staining overlaps (yellow staining in $\mathbf{I}$ and $\mathbf{o}$ ). Ezrin is also expressed in proximal tubules (k, arrowheads) where NHERF1 (g) but not NHERF2 (I) is detected. Rat kidneys were processed for semithin $(0.5 \mu \mathrm{m})$ cryosectioning, followed by double staining with mAbs anti-PC (5A) or anti-ezrin (3C12) and polyclonal anti-NHERF1 or anti-NHERF2, as described in Methods. Bars: (a-c and g-I) $20 \mu \mathrm{m}$; (d-f and $\mathbf{m}-\mathbf{o}) 5 \mu \mathrm{m}$.

end we carried out immunoprecipitation on glomerular lysates with anti-PC, anti-NHERF2, or anti-ezrin, followed by immunoblotting as above. The effects of the above reagents were checked by routine transmission EM (Figure 7).

After immunoprecipitation with anti-PC, there was a significant reduction $(70-90 \%)$ in the amount of NHERF2 in the precipitates in SA-treated (Figure 8a, lane 5) and PS-treated (Figure 8a, lane 6) glomeruli. The interaction was slightly reduced $(\sim 30 \%)$ in PAN $3 \mathrm{~d}$ glomeruli and unchanged in PAN 10d glomeruli (Figure 8a, lanes 3 and 4). The amount of ezrin was also dramatically reduced (by $\sim 90 \%$ ) in SA-treated (Figure 8a, lane 5) and PS-treated (Figure 8a, lane 6) glomeruli, only slightly reduced in PAN 3d glomeruli, and unchanged in PAN 10d glomeruli (Figure 8a, lanes 3 and 4). Interestingly, heparin perfusion of PS-treated rats (Figure 8a, lane 7) reversed the effect. These results indicate that the PCNHERF2 interaction is disrupted after SA or PS treatment, but interaction is restored by heparin treatment.

When immunoprecipitation was carried out with anti-ezrin, the results were similar in that there was a 

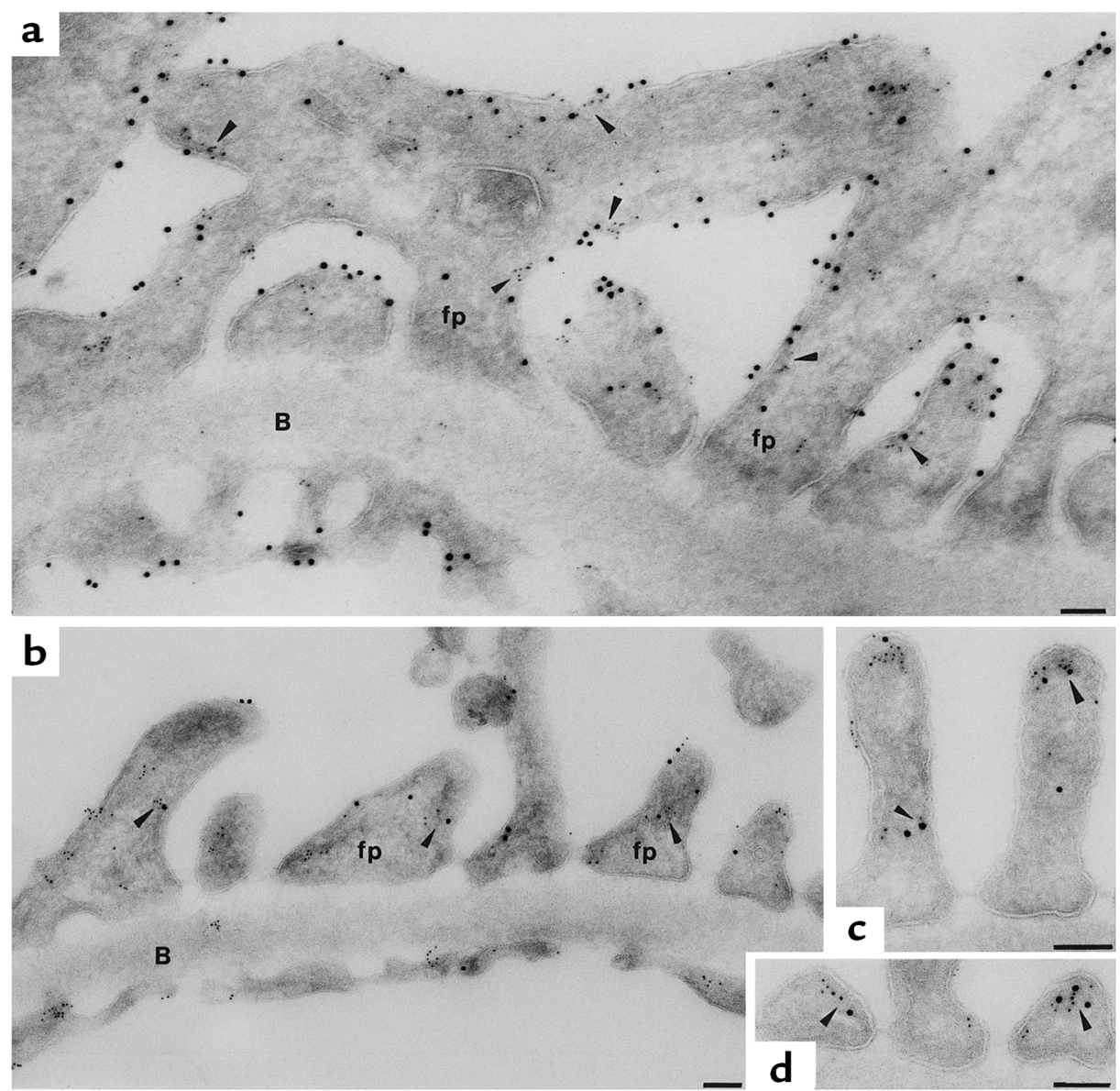

\section{Figure 5}

Immunogold localization of NHERF2 in the glomerulus. (a) Double labeling for $P C$ and NHERF2 in the rat glomerulus. PC (10 $\mathrm{nm}$ gold) and NHERF2 (5 nm gold) colocalize along the apical PM of foot processes (fp) above the slit diaphragms and along the cell membrane of the cell bodies of GECs (arrowheads). PC is also localized along the luminal surface of the glomerular endothelium (En), but NHERF2 is absent. No labeling for NHERF2 is seen at the base of the foot processes facing the GBM (B). (b, c, and d) Double labeling for ezrin and NHERF2 in rat glomeruli. Ezrin (10 $\mathrm{nm}$ gold) and NHERF2 (5 nm gold) are colocalized mainly along the apical PM of foot processes (arrowheads) but are not found along the basal surface of the foot processes below the level of the slit diaphragms. Ultrathin cryosections were doubly incubated with polyclonal anti-NHERF2 and either anti-PC (5A) or anti-ezrin (3C12) IgG followed by 5 - or $10-\mathrm{nm}$ gold goat anti-rabbit or anti-mouse IgG conjugates as described in Methods. Bars, $0.1 \mu \mathrm{m}$. dramatic reduction in the $\mathrm{PC}$ found in the precipitates in the SA-treated (Figure 8b, lane 5) and PS-treated (Figure $8 \mathrm{~b}$, lane 6) glomeruli and a slight reduction in PAN $3 \mathrm{~d}$ glomeruli (Figure 8b, lane 3). However, there was no change in the amount of NHERF2 that coprecipitated under any of those conditions (Figure 8b, bottom panel), indicating that the NHERF2-ezrin interaction is largely unchanged among these experimental groups.

We conclude that the PS and SA treatments disrupt PC-NHERF2 interaction, but ezrin-NHERF2 interaction is maintained under all these conditions.

Dissociation of PC/NHERF2/ezrin from the actin cytoskeleton after PAN and SA treatments. We showed recently by differential extraction that PC cosediments with actin filaments (15), which are resistant to mild detergent extraction. To further investigate the interactions of PC under conditions known to disrupt GEC shape, we carried out sequential extraction of glomeruli to find out if the PC/NHERF2/ezrin complex dissociates from the actin cytoskeleton of GECs after any of these treatments. Sequential detergent extraction partitions cellular proteins into functionally distinct compartments $(27,38,39)$ and preserves cytoskeletal integrity and cytoskeletal-noncytoskeletal interactions (38). Isolated glomeruli from normal, PS-, SA-, and PANtreated rats were sequentially solubilized in $0.5 \%$ Tri- ton X-100 (TX) and RIPA lysis buffer and separated into Triton X-100 soluble (TXS), RIPA soluble (RIPA), and RIPA insoluble fractions (Ppt), followed by immunoblotting.

PC, NHERF2, and ezrin distributed in all three fractions (Figure 9; percentages approximate) in normal glomeruli with $75 \%$ of the total PC in TXS, $20 \%$ in RIPA, and $5 \%$ in Ppt, and 55\% of the NHERF2 in TXS, $35 \%$ in RIPA, and $10 \%$ in Ppt. SA and PAN, but not PS, treatments resulted in a significant increase in the detergent solubility of PC and NHERF2 (Figure 9). In SA-treated glomeruli PC distributed $95 \%$ in TXS and $5 \%$ in RIPA, and NHERF2 distributed $75 \%$ in TXS and $25 \%$ in RIPA. In PAN rats PC distributed $95 \%$ in TXS and $5 \%$ in RIPA, and NHERF 2 distributed $70 \%$ in TXS and $30 \%$ in RIPA. Thus none of the PC or NHERF was found in the insoluble fraction, indicating that none remained associated with actin after SA or PAN treatments. The distribution of actin was unchanged among these experimental groups with $15 \%$ each in TXS and RIPA and 70\% in Ppt.

In the case of ezrin it was distributed in all three fractions after sequential extraction of normal glomeruli, that is, $50 \%$ in TXS, $30 \%$ in RIPA, and 20\% in Ppt. In SA-treated rats it distributed $75 \%$ in TXS, $20 \%$ in RIPA, and 5\% in Ppt, and in PAN rats it distributed 65\% in TXS, 30\% in RIPA, and 5\% in Ppt 
a

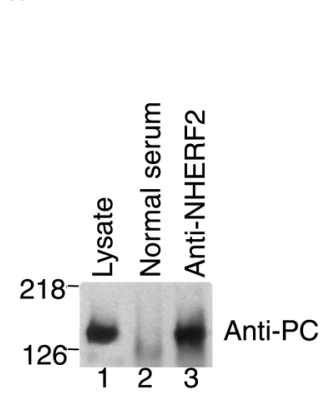

b

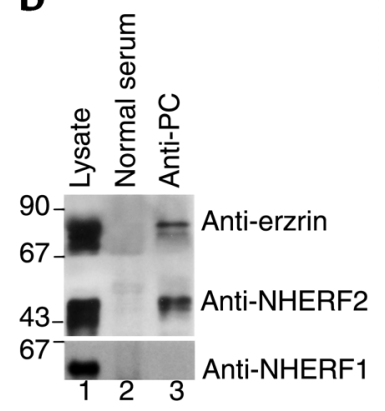

(Figure 9). Thus in rats treated with SA and PAN, the ezrin remaining in the insoluble fraction was significantly decreased.

Since activated ezrin, cross-links actin filaments to the PM with concomitant C-terminal threonine (T567) phosphorylation (40), we examined whether the above treatments alter the level of threonine phosphorylated ezrin. Phosphorylated ezrin was present only in Ppt in all experimental groups; however, the level of phosphorylated ezrin was reduced dramatically in SA-treated $(\sim 90 \%)$ and PAN-treated $(\sim 80 \%)$ glomeruli (Figure 9). These results suggest that SA and PAN treatments lead to dephosphorylation of ezrin and its dissociation from the actin cytoskeleton. Based on these results, together with those from coimmunoprecipitation assays, we conclude that the pathological changes found in GECs of all three experimental groups are associated with disruption of PC/NHERF2/ezrin complexes and dissociation of PC from the actin cytoskeleton.

\section{Discussion}

We have shown recently that the biochemical properties of the ectodomain of PC are responsible for keeping the filtration slits open between adjacent foot processes (14) and that the cytoplasmic domain of PC associates with the actin cytoskeleton through complex formation with ezrin (15), an ERM protein. These observations suggested that PC may contribute to foot process formation and maintenance through association with the actin cytoskeleton. In the present study, we have shown that NHERF2, a PDZ domain-containing protein, interacts directly with PC, and that PC, NHERF2, and ezrin colocalize in GECs and form a multimeric protein complex. We further report the striking finding that treat-

\section{Figure 7}

Morphologic alterations seen in PAN-treated kidney. (a) Portion of a glomerular capillary from a normal, untreated rat showing the typical organization of the foot processes (fp) of the glomerular epithelium. (b) Portion of a capillary from a PAN nephrotic rat showing disruption of the foot process organization of GECs (Ep) and loss of the filtration slits between foot processes. BM, basement membrane; En, endothelium. Bar, $0.5 \mu \mathrm{m}$.

\section{Figure 6}

PC, NHERF2, and ezrin form a coprecipitable complex in rat glomeruli. (a) Immunoprecipitation was carried out on normal glomerular lysates with polyclonal anti-NHERF2 (lane 3) or control (lane 2) serum followed by immunoblotting with monoclonal antiPC (5A). PC is seen in NHERF2 precipitates. Lane 1: $10 \mu \mathrm{g}$ glomerular lysate. (b) A similar immunoprecipitation was carried out with anti-PC $m A b s(1 A+5 A)$ followed by blotting with polyclonal antiezrin, anti-NHERF1, or anti-NHERF2. Both NHERF2 and ezrin coprecipitate with PC, but NHERF1 is not detected (lane 3). Neither PC nor NHERF2 are detected in control serum precipitates (lane 2). Lane 1: $10 \mu \mathrm{g}$ glomerular lysate. The experiment was performed twice with comparable results.

ments that affect foot process organization of GECs disrupt the PC/NHERF2/ezrin/actin cytoskeleton association.

NHERF2, also called E3KARP, was initially identified by two-hybrid screening using the C-terminal, cytoplasmic tail of NHE3 (28). Two isotypes of NHERF, NHERF1 (EBP50) and NHERF2 (E3KARP), have been identified, which have $48 \%$ amino acid sequence identity and two PDZ domains and an ERM-binding region in common $(28,34)$. Both NHERF1 and NHERF2 are thought to be regulators of NHE3 because they are required for the cAMP-dependent inhibition of NHE3.

Studies of the molecular interactions of proteins with PDZ domains have shown that distinct PDZ domains interact with specific $\mathrm{C}$-termini carrying the consensus sequence $\mathrm{X}(\mathrm{S} / \mathrm{T}) \mathrm{X}(\mathrm{V} / \mathrm{L} / \mathrm{I})$ (31). NHERF2 interacts through its PDZ domains with the last four $\mathrm{C}$-terminal residues of several proteins, including the $\beta_{2}$-adrenergic receptor (41), the cystic fibrosis transmembrane conductance regulator (CFTR) (23), phospholipase C$\beta 3$ (42), and NHE3 (36). In all cases the interaction is believed to link membrane proteins with the actin cytoskeleton through ezrin (29).

In this study we identified the NHERF2-binding site on $\mathrm{PC}$ as the $\mathrm{C}$-terminal PDZ-binding motif DTHL, the last four C-terminal residues of $\mathrm{PC}$, because deletion of
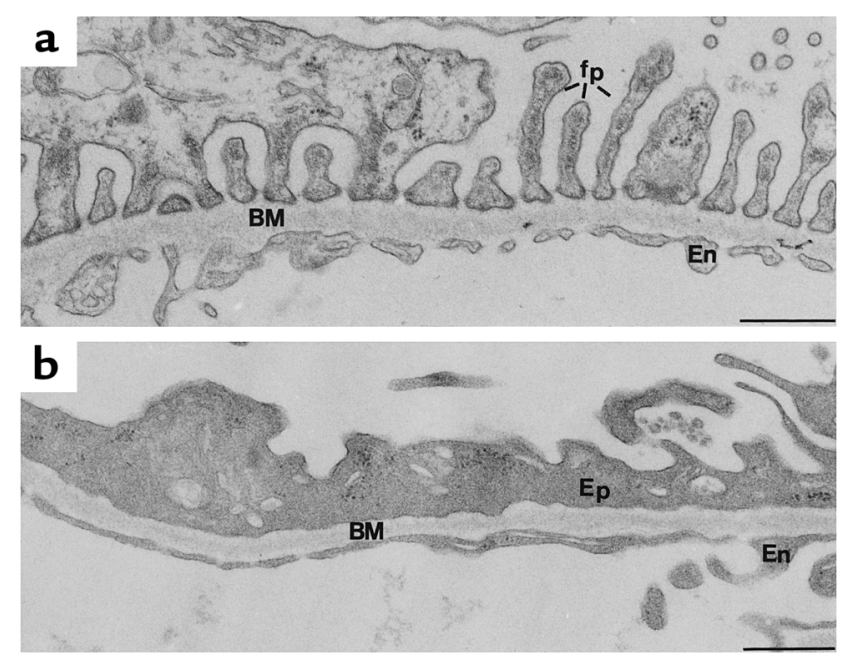

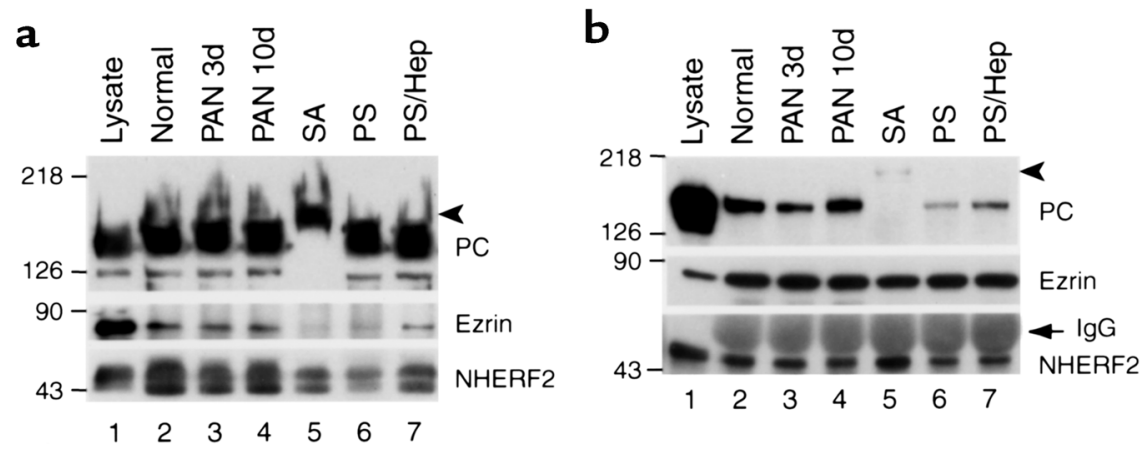

\section{Figure 8}

Disruption of PC/NHERF2/ezrin complexes in glomeruli from PAN-, SA-, and PS-treated rats. (a) Immunoprecipitation with anti-PC followed by immunoblotting for NHERF2 and ezrin as for Figure 6. The amount of ezrin and NHERF2 that coprecipitates with PC is reduced significantly in SA-treated (lane 5) and PS-treated (lane 6), but not in PAN-treated, glomeruli (lanes 3 and 4) compared with normal controls (lane 2). The amount of ezrin and NHERF2 that coprecipitates is restored to normal after heparin (Hep) perfusion of PS-treated rats (lane 7). Lane 1: $10 \mu \mathrm{g}$ glomerular lysate. (b) When immunoprecipitation is carried out with anti-ezrin followed by immunoblotting for NHERF2 and PC, a similar reduction is seen in the amount of PC that coprecipitates with ezrin in SA (lane 5) and PS (lane 6) treated rats but not in PAN nephrotic rats (lanes 3 and 4). No change is seen in the amount of NHERF2 coprecipitated (bottom panel). Glomerular lysates from normal, PAN 3$d$, and PAN10d, SA- and PS-treated rats were immunoprecipitated with anti-PC (1A + 5A) mAb (a) or polyclonal anti-ezrin (b) followed by immunoblotting as in Figure 6. Lane 1: $10 \mu \mathrm{g}$ control lysate. Arrowheads indicate desialylated PC, which has a slower mobility than intact PC (lane 5). The arrow indicates IgG heavy chain. The experiment was performed twice with comparable results.

DTHL prevented its interaction with NHERF2. Results of GST pull-down assays using fragments of NHERF2 showed that only PDZ2 interacts with the PC tail.

We have also shown that NHERF2 colocalizes and interacts with PC in GECs in situ. NHERF1 can also associate with $\mathrm{PC}$ in vitro, but it does not colocalize with $\mathrm{PC}$ in the kidney in vivo. NHERF1 is expressed mainly in proximal tubules (32) where it interacts with NHE3 (43). Thus both NHERF proteins are found in the kidney, but they are expressed in different cell types and hence are presumably involved in separate cellular functions.

Our findings demonstrate that PC and NHERF2 form a multimeric complex with ezrin in vivo and that these proteins are present at the apical PM of GECs. In analogy to the interactions of NHERF with CFTR, NHE3, and the $\beta_{2}$-adrenergic receptor $(29,30)$, our findings suggest that NHERF2 acts as a scaffold to connect PC to the cytoskeleton through association a

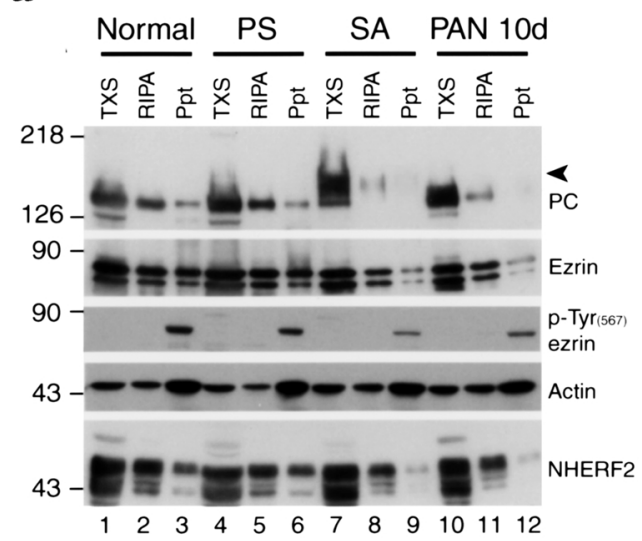

b
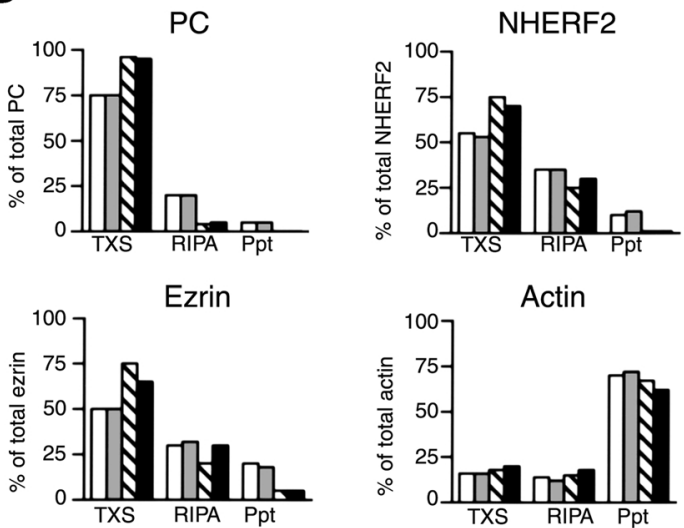

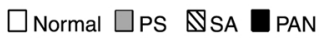

Figure 9

Dissociation of PC/NHERF2/ezrin complexes from the cytoskeleton in glomeruli from SA- and PAN-treated rats. (a) Immunoblot of normal, PS, SA-, or PAN-treated (10d) rats. In normal glomeruli, PC, NHERF2, and ezrin distribute in all three fractions - that is, Triton X-100 soluble (TXS), RIPA soluble (RIPA), and RIPA insoluble (Ppt). In SA- and PAN-, but not PS-treated glomeruli, there is a significant increase in the amount of PC, NHERF2, and ezrin found in the Triton X-100 soluble (TXS) fraction. Actin is also distributed in all three fractions, but its pattern of distribution is unchanged among these experimental groups. C-terminal threonine phosphorylated ezrin [ezrin, $\mathrm{pTyr}(567)]$ is present only in the insoluble precipitate (Ppt) (lanes 3, 6, 9, and 12) under all conditions. The level of phosphorylated ezrin is significantly reduced in SA- and PAN-treated glomeruli. Isolated glomeruli from normal, PS-, SA-, and PAN-treated (10d) rats were solubilized sequentially with $0.5 \%$ Triton X-100 and RIPA lysis buffer and separated into Triton X-100-soluble (TXS), RIPA-soluble (RIPA), and RIPA-insoluble fractions (Ppt). Equal volumes of these fractions were immunoblotted with appropriate Ab's. The arrowhead indicates the position of desialylated PC (lane 7). The experiment was performed twice with comparable results. (b) Quantification of protein bands shown in a was done by densitometry as described in Methods. 

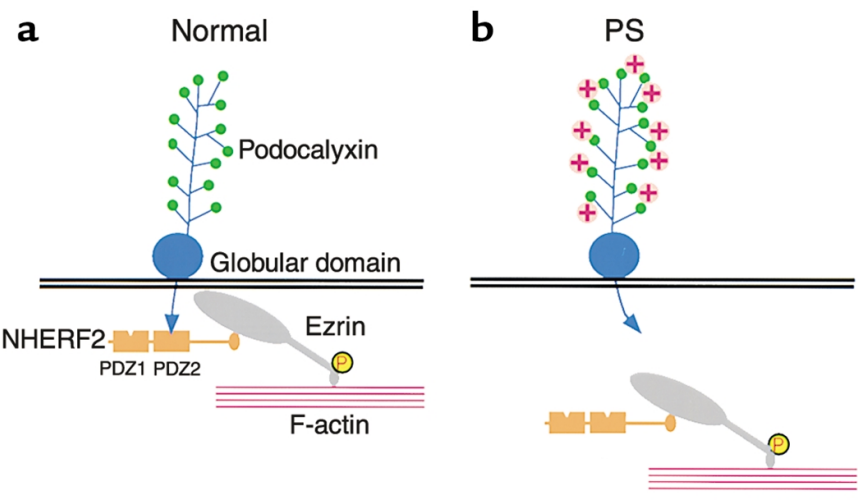

c

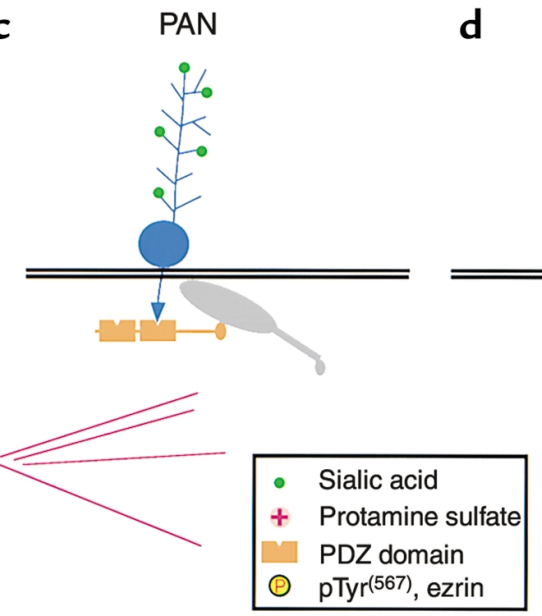

SA
Figure 10

Models for the sites of disruption of the $\mathrm{PC} / \mathrm{NHERF}$ /ezrin complex with the actin cytoskeleton in pathological conditions associated with changes in foot process organization. (a) Normal glomerulus. PC binds to the second PDZ domain (PDZ2) of NHERF2 through its C-terminal PDZ-binding motif along the apical region of GECs. NHERF2 binds to the $\mathrm{N}$-terminus of phosphorylated (unmasked) ezrin through its C-terminal ERMbinding region. Unmasked phosphorylated (P) ezrin links the complex to the actin cytoskeleton through its C-terminal actin-binding site. (b) Protamine sulfate (PS) treatment. Neutralization of the negative charge on the extracellular domain of PC by PS disrupts the binding of the cytoplasmic tail of PC to NHERF2. (c) PAN (10d) treatment. The $\mathrm{PC} / \mathrm{NHERF} 2 /$ ezrin complex remains intact, but ezrin dissociates from the actin cytoskeleton with a concomitant decrease in the level of phosphorylated ezrin. (d) Sialidase (SA) treatment. When sialic acid is removed from PC by SA digestion, the complex is disrupted in two places: PC dissociates from NHERF2 and ezrin dissociates from the actin cytoskeleton with a concomitant reduction in the level of phosphorylated ezrin. with ezrin as diagramed in Figure 10. In the normal glomerulus (Figure 10a), PC binds to PDZ2 of NHERF2 through its C-terminus, NHERF2 binds to the N-terminal half of unmasked ezrin through its Cterminal ERM-binding region (36), and C-terminal threonine phosphorylated ezrin links the complex with the actin cytoskeleton through its C-terminal actin binding site (40). This model is supported by our findings indicating that a significant portion of PC and ezrin cosediment with actin following mild detergent extraction, whereas both proteins are found in the soluble fraction after depolymerization of filamentous actin (15). Thus NHERF2 and ezrin serve as intermediates linking PC to the cortical actin cytoskeleton. The association of PC with a cytoskeletal complex could serve to anchor PC to specific microdomains of the apical membrane or determine its residence time at the cell surface.

We further show here that dissociation of PC from the actin cytoskeleton occurs in pathological conditions associated with changes in GEC shape. In PStreated rats (Figure 10b), the PC-NHERF2 interaction is uncoupled in response to neutralization of the extracellular domain. In PAN-treated rats (Figure 10c), the ezrin-actin interaction is uncoupled with a concomi- tant decrease in phosphorylated ezrin. In SA-treated rats (Figure 10d), phosphorylated ezrin is also reduced and both the PC-NHERF2 and ezrin-actin interactions are disrupted due to removal of sialic acid.

In PAN rats the morphologic alterations of GECs are associated with a reduction in the sialic acid content of PC to one-third of normal (44), and neutralization of the glomerular surface charge in normal rats by perfusion of PS or removal of sialic acid by SA digestion causes a narrowing of the filtration slits, effacement of the foot processes $(8,9,11,45)$, and redistribution and tyrosine phosphorylation of the junctional protein, ZO-1 $(10,16)$. These findings lead us to propose a mechanism through which the loss of the negative charge at the surface of GECs, carried for the most part by PC, changes the conformation of PC and transduces an intracellular signal that affects the PC-cytoskeleton connection and alters GEC morphology.

Quite recently, it has been found that deletion of the PC gene in the mouse results in neonatal lethality and failure to form foot processes in the homozygote (46), which is in keeping with our conclusion that association of PC with the cytoskeleton through adaptor proteins is essential for normal development of foot processes. Mutations in several other human and murine genes, 
including nephrin, $\mathrm{CD} 2 \mathrm{AP}$, podocin, $\alpha$-actinin $4, \alpha 3 \beta 1$ integrin, and laminin $\beta 2$, also lead to disruption of podocyte architecture and the nephrotic syndrome. Mutations in the nephrin gene, a component of the slit diaphragm that attaches neighboring foot processes to one another, occur in humans with congenital nephrotic syndrome (Finnish type), where there is also failure to form foot processes and massive proteinuria (47). A similar phenotype is seen in mice with knockout of CD2AP, which may link nephrin to the actin cytoskeleton (48). Interestingly, deletion of $\alpha 3$ integrin, an adhesion molecule that attaches the foot processes to the GBM and its potential binding partner in the GBM, laminin $\beta 2$, also results in changes in foot processes and proteinuria (49, 50). Genetic analysis of families with proteinuria leading to glomerulosclerosis has identified several other mutated proteins involved in cytoskeletal-basement membrane ( $\alpha$-actinin 4 ) and cytoskeleton-PM (podocin) interactions in GECs $(51,52)$. Presumably all these mutations result in defective maturation of glomeruli.

Alterations in the organization of foot processes and proteinuria can also be induced by in vivo infusion of mAb's against several membrane proteins of GECs, including those against nephrin 5-1-6 (53), podoplanin (54), and aminopeptidase A (55). Thus targeting by gene disruption or $\mathrm{Ab}$ injection of molecules responsible for maintaining the cytoskeleton and cell shape, particularly those that affect the relationship between neighboring foot processes and the foot processes and the GBM, result in major changes in the structure and function of GECs. The precise mechanisms involved have not been established except in the case of PC.

Rearrangement of GEC organization is often associated with increased glomerular permeability and proteinuria. It should be remembered, however, that in humans with nephrotic syndrome, as well as rats with PAN nephrosis, foot process changes go hand in hand with changes in intercellular junctions $(16,37)$ and the GBM. There is thinning of the GBM (56) and disruption of the anionic sites (57) composed of heparan sulfate proteoglycans in the GBM (58). Moreover, the effects of PS and SA treatments are not restricted to GECs, as they also result in neutralization of anionic sites and removal of sialic acid from the GBM as well as from the GEC surface. Thus there is a close interplay between GEC shape, junctional organization, and integrity of the GBM.

Our demonstration that the cytoplasmic domain of $\mathrm{PC}$ is linked to the actin cytoskeleton through NHERF/ezrin complexes, taken together with our previous work showing the extracellular domain contributes to the anti-adhesion function of $\mathrm{PC}$, indicates that $\mathrm{PC}$ is a major player in maintaining foot process structure in vivo. Further investigations are required to determine what signal(s) are involved in the maintenance or disruption of the PC/NHERF2/ezrin complex in GECs and should shed light on the molecular mechanisms responsible for the changes in foot process structure characteristic of the nephrotic syndrome.

PC is expressed not only in GECs but also in vascular endothelia; however, ezrin is not present in either peritubular capillaries or in the glomerular endothelium. Other members of the ERM family such as moesin, which is known to be expressed in endothelial cells (59), may be involved in PC-cytoskeletal association in these cells.

\section{Acknowledgments}

The rat NHERF2 cDNA sequence reported in this paper has been deposited in the GenBank database with accession number AF 294257. This research was supported by grant DK-17724 to M.G. Farquhar from the NIH.

1. Farquhar, M.G., Vernier, R.L., and Good, R.A. 1957. Study of familial nephrosis. II. Glomerular changes observed with the electron microscope. Am. J. Pathol. 33:791-817.

2. Farquhar, M.G., Vernier, R.L., and Good, R.A. 1957. An electron microscopic study of the glomerulus in nephrosis, glomerulonephritis and lupus erythematosus. J. Exp. Med. 106:649-660.

3. Gibson, I.W., and More, I.A. 1998. Glomerular pathology: recent advances. J. Pathol. 184:123-129.

4. Tejani, A., and Ingulli, E. 1995. Current concepts of pathogenesis of nephrotic syndrome. Contrib. Nephrol. 114:1-5.

5. Smoyer, W.E., and Mundel, P. 1998. Regulation of podocyte structure during the development of nephrotic syndrome. J. Mol. Med. 76:172-183.

6. Mohos, S.C., and Skoza, L. 1969. Glomerular sialoprotein. Science. 164:1519-1521.

7. Michael, A.F., Blau, E., and Vernier, R.L. 1970. Glomerular polyanion. Alteration in aminonucleoside nephrosis. Lab. Invest. 23:649-657.

8. Seiler, M.W., Rennke, H.G., Venkatachalam, M.A., and Cotran, R.S. 1977. Pathogenesis of polycation-induced alterations of glomerular epithelium. Lab. Invest. 36:48-61.

9. Kerjaschki, D. 1978. Polycation-induced dislocation of slit diaphragms and formation of cell junctions in rat kidney glomeruli: the effects of low temperature, divalent cations, colchicine, and cytochalasin B. Lab. Invest. 39:430-440.

10. Kurihara, H., Anderson, J.M., Kerjaschki, D., and Farquhar, M.G. 1992. The altered glomerular filtration slits seen in puromycin aminonucleoside nephrosis and protamine sulfate-treated rats contain the tight junction protein ZO-1. Am. J. Pathol. 141:805-816.

11. Andrews, P.M. 1979. Glomerular epithelial alterations resulting from sialic acid surface coat removal. Kidney Int. 15:376-385.

12. Gelberg, H., Healy, L., Whiteley, H., Miller, L.A., and Vimr, E. 1996. In vivo enzymatic removal of $\alpha 2 \rightarrow 6$-linked sialic acid from the glomerular filtration barrier results in podocyte charge alteration and glomerular injury. Lab Invest. 74:907-920.

13. Kerjaschki, D., Sharkey, D.J., and Farquhar, M.G. 1984. Identification and characterization of podocalyxin: the major sialoprotein of the renal glomerular epithelial cell. J. Cell Biol. 98:1591-1596.

14. Takeda, T., Go, W.Y., Orlando, R.A., and Farquhar, M.G. 2000. Expression of podocalyxin inhibits cell-cell adhesion and modifies junctional properties in Madin-Darby canine kidney cells. Mol. Biol. Cell. 11:3219-3232.

15. Orlando, R.A., et al. 2001. The glomerular epithelial cell anti-adhesin, podocalyxin, associates with the actin cytoskeleton through interactions with ezrin. J. Am. Soc. Nephrol. In Press.

16. Kurihara, H., Anderson, J.M., and Farquhar, M.G. 1995. Increased Tyr phosphorylation of $\mathrm{ZO}-1$ during modification of tight junctions between glomerular foot processes. Am. J. Physiol. 268:F514-F524.

17. Mangeat, P., Roy, C., and Martin, M. 1999. ERM proteins in cell adhesion and membrane dynamics. Trends Cell Biol. 9:187-192.

18. Bretscher, A. 1999. Regulation of cortical structure by the ezrin-radixinmoesin protein family. Curr. Opin. Cell Biol. 11:109-116.

19. Schnabel, E., Dekan, G., Miettinen, A., and Farquhar, M.G. 1989. Biogenesis of podocalyxin -the major glomerular sialoglycoprotein- in the newborn rat kidney. Eur. J. Cell Biol. 48:313-326.

20. Whiteside, C.I., Cameron, R., Munk, S., and Levy, J. 1993. Podocytic cytoskeletal disaggregation and basement-membrane detachment in puromycin aminonucleoside nephrosis. Am. J. Pathol. 142:1641-1653.

21. Altschul, S.F., Gish, W., Miller, W., Myers, E.W., and Lipman, D.J. 1990. Basic local alignment search tool. J. Mol. Biol. 215:403-410.

22. Miettinen, A., Dekan, G., and Farquhar, M.G. 1990. Monoclonal antibodies against membrane proteins of the rat glomerulus. Immunochemical specificity and immunofluorescence distribution of the antigens. Am. J. Pathol. 137:929-944.

23. Sun, F., et al. 2000. E3KARP mediates the association of ezrin and protein kinase A with the cystic fibrosis transmembrane conductance regulator in airway cells. J. Biol. Chem. 275:29539-29546. 
24. McCaffery, J.M., and Farquhar, M.G. 1995. Localization of GTPases by indirect immunofluorescence and immunoelectron microscopy. Methods Enzymol. 257:259-279.

25. Kanwar, Y.S., and Farquhar, M.G. 1980. Detachment of endothelium and epithelium from the glomerular basement membrane produced by kidney perfusion with neuraminidase. Lab Invest. 42:375-384.

26. Ryan, G.B., and Karnovsky, M.J. 1975. An ultrastructural study of the mechanisms of proteinuria in aminonucleoside nephrosis. Kidney Int. 8:219-232.

27. Lenk, R., Ransom, L., Kaufmann, Y., and Penman, S. 1977. A cytoskeletal structure with associated polyribosomes obtained from HeLa cells. Cell. 10:67-78.

28. Yun, C.H., et al. 1997. cAMP-mediated inhibition of the epithelial brush border $\mathrm{Na}^{+} / \mathrm{H}^{+}$exchanger, NHE3, requires an associated regulatory protein. Proc. Natl. Acad. Sci. USA. 94:3010-3015.

29. Bretscher, A., Chambers, D., Nguyen, R., and Reczek, D. 2000. ERM-Merlin and EBP50 protein families in plasma membrane organization and function. Annu. Rev. Cell Dev. Biol. 16:113-143.

30. Fanning, A.S., and Anderson, J.M. 1999. Protein modules as organizers of membrane structure. Curr. Opin. Cell Biol. 11:432-439.

31. Songyang, Z., et al. 1997. Recognition of unique carboxyl-terminal motifs by distinct PDZ domains. Science. 275:73-77.

32. Breton, S., et al. 2000. The $\mathrm{B} 1$ subunit of the $\mathrm{H}^{+} \mathrm{ATP}$ ase is a PDZ domain binding protein. Colocalization with NHE-RF in renal B-intercalated cells. J. Biol. Chem. 275:18219-18224.

33. Wade, J.B., Welling, P.A., Donowitz, M., Shenolikar, S., and Weinman, E.J. 2001. Differential renal distribution of NHERF isoforms and their colocalization with NHE3, ezrin, and ROMK. Am. J. Physiol. Cell Physiol. 280:C192-C198

34. Reczek, D., Berryman, M., and Bretscher, A. 1997. Identification of EBP50: A PDZ-containing phosphoprotein that associates with members of the ezrin-radixin-moesin family. J. Cell Biol. 139:169-179.

35. Lamprecht, G., Weinman, E.J., and Yun, C.H. 1998. The role of NHERF and E3KARP in the cAMP-mediated inhibition of NHE3. J. Biol. Chem. 273:29972-29978.

36. Yun, C.H., Lamprecht, G., Forster, D.V., and Sidor, A. 1998. NHE3 kinase A regulatory protein E3KARP binds the epithelial brush border $\mathrm{Na}^{+} / \mathrm{H}^{+}$ exchanger NHE3 and the cytoskeletal protein ezrin. J. Biol. Chem. 273:25856-25863.

37. Caulfield, J.P., Reid, J.J., and Farquhar, M.G. 1976. Alterations of the glomerular epithelium in acute aminonucleoside nephrosis. Evidence for formation of occluding junctions and epithelial cell detachment. Lab Invest. 34:43-59.

38. Fey, E.G., Capco, D.G., Krochmalnic, G., and Penman, S. 1984. Epithelial structure revealed by chemical dissection and unembedded electron microscopy. J. Cell Biol. 99(Suppl.):203s-208s.

39. Ramsby, M.L., Makowski, G.S., and Khairallah, E.A. 1994. Differential detergent fractionation of isolated hepatocytes: biochemical, immunochemical and two-dimensional gel electrophoresis characterization of cytoskeletal and noncytoskeletal compartments. Electrophoresis. 15:265-277.

40. Matsui, T., et al. 1998. Rho-kinase phosphorylates $\mathrm{COOH}$-terminal threonines of ezrin/radixin/moesin (ERM) proteins and regulates their headto-tail association. J. Cell Biol. 140:647-657.

41. Hall, R.A., et al. 1998. A C-terminal motif found in the beta2-adrenergic receptor, $\mathrm{P} 2 \mathrm{Y} 1$ receptor and cystic fibrosis transmembrane conductance regulator determines binding to the $\mathrm{Na}^{+} / \mathrm{H}^{+}$exchanger regulatory factor family of PDZ proteins. Proc. Natl. Acad. Sci. USA. 95:8496-8501.

42. Hwang, J.I., et al. 2000. Regulation of phospholipase C-beta 3 activity by $\mathrm{Na}^{+} / \mathrm{H}^{+}$exchanger regulatory factor 2. J. Biol. Chem. 275:16632-16637.

43. Amemiya, M., et al. 1995. Expression of NHE-3 in the apical membrane of rat renal proximal tubule and thick ascending limb. Kidney Int. 48:1206-1215.

44. Kerjaschki, D., Vernillo, A.T., and Farquhar, M.G. 1985. Reduced sialylation of podocalyxin -the major sialoprotein of the rat kidney glomerulus- in aminonucleoside nephrosis. Am. J. Pathol. 118:343-349.

45. Kanwar, Y.S., and Rosenzweig, L.J. 1982. Altered glomerular permeability as a result of focal detachment of the visceral epithelium. Kidney Int. 21:565-574.

46. Duhme, C., McNagny, K., Killen, P., and Kershaw, D. 1999. Homozygous disruption of the murine podocyte/MEP21 gene leads to disordered glomerular structure and neonatal death. J. Am. Soc. Nephrol. 10:554a. (Abstr.)

47. Tryggvason, K. 1999. Unraveling the mechanisms of glomerular ultrafiltration: nephrin, a key component of the slit diaphragm. J. Am. Soc. Nephrol. 10:2440-2445.

48. Shih, N.Y., et al. 1999. Congenital nephrotic syndrome in mice lacking CD2-associated protein. Science. 286:312-315.

49. Noakes, P.G., et al. 1995. The renal glomerulus of mice lacking slaminin/laminin beta 2: nephrosis despite molecular compensation by laminin beta 1. Nat. Genet. 10:400-406.

50. Kreidberg, J.A., et al. 1996. Alpha 3 beta 1 integrin has a crucial role in kidney and lung organogenesis. Development. 122:3537-3547.

51. Kaplan, J.M., et al. 2000. Mutations in ACTN4, encoding alpha-actinin-4, cause familial focal segmental glomerulosclerosis. Nat. Genet. 24:251-256.

52. Boute, N., et al. 2000. NPHS2, encoding the glomerular protein podocin, is mutated in autosomal recessive steroid-resistant nephrotic syndrome. Nat. Genet. 24:349-354.

53. Orikasa, M., Matsui, K., Oite, T., and Shimizu, F. 1988. Massive proteinuria induced in rats by a single intravenous injection of a monoclonal antibody. J. Immunol. 141:807-814.

54. Matsui, K., Breiteneder-Geleff, S., and Kerjaschki, D. 1998. Epitope-specific antibodies to the $43-\mathrm{kD}$ glomerular membrane protein podoplanin cause proteinuria and rapid flattening of podocytes. J. Am. Soc. Nephrol. 9:2013-2026.

55. Assmann, K.J., van Son, J.P., Dijkman, H.B., and Koene, R.A. 1992. A nephritogenic rat monoclonal antibody to mouse aminopeptidase A. Induction of massive albuminuria after a single intravenous injection. $J$. Exp. Med. 175:623-635.

56. Caulfield, J.P., and Farquhar, M.G. 1975. The permeability of glomerular capillaries of aminonuceoside nephrotic rats to graded dextrans. $J$. Exp. Med. 142:61-83.

57. Caulfield, J.P., and Farquhar, M.G. 1978. Loss of anionic sites from the glomerular basement membrane in aminonucleoside nephrosis. $L a b$. Invest. 39:505-512.

58. Kanwar, Y.S., and Farquhar, M.G. 1979. Isolation of glycosaminoglycans (heparan sulfate) from glomerular basement membranes. Proc. Natl. Acad. Sci. USA. 76:4493-4497.

59. Berryman, M., Franck, Z., and Bretscher, A. 1993. Ezrin is concentrated in the apical microvilli of a wide variety of epithelial cells whereas moesin is found primarily in endothelial cells. J. Cell Sci. 105:1025-1043. 